\title{
LOWER BOUNDS AND THE HARDNESS OF COUNTING PROPERTIES
}

\author{
Lane A. Hemaspaandra and Mayur Thakur \\ Department of Computer Science \\ University of Rochester, Rochester NY 14627, USA \\ $\{$ lane, thakur\}@cs.rochester.edu
}

\begin{abstract}
Rice's Theorem states that all nontrivial language properties of recursively enumerable sets are undecidable. Borchert and Stephan [BS00] started the search for complexity-theoretic analogs of Rice's Theorem, and proved that every nontrivial counting property of boolean circuits is UP-hard. Hemaspaandra and Rothe [HROO] improved the UP-hardness lower bound to $\mathrm{UP}_{O(1)}$-hardness. The present paper raises the lower bound for nontrivial counting properties from $\mathrm{UP}_{O(1)}$-hardness to FewPhardness, i.e., from constant-ambiguity nondeterminism to polynomialambiguity nondeterminism. Furthermore, we prove that this lower bound is rather tight with respect to relativizable techniques, i.e., no relativizable technique can raise this lower bound to FewP- $\leq_{1-t t}^{p}$-hardness. We also prove a Rice-style theorem for NP, namely that every nontrivial language property of NP sets is NP-hard.
\end{abstract}

Keywords: ambiguity-bounded computation, boolean circuits, computational complexity, counting properties, lower bounds, Rice's Theorem.

\section{Introduction}

The relationship between languages and the machines used to recognize them plays an important role in both computability theory and complexity theory. Languages are semantic objects with which computability and complexity theories deal. Machines are syntactic objects used to describe the languages.

Rice's Theorem ([Ric53], see also [Ric56]) links, in a rather thrilling and broad way, these semantic and syntactic objects. Rice's Theorem says that for any language class $\mathcal{C}, \emptyset \subsetneq \mathcal{C} \subsetneq \mathrm{RE}$, the set of all machines whose languages belong to $\mathcal{C}$ is highly noncomputable, in particular is RE- $\leq_{m}$-hard or coRE$\leq_{m}$-hard. Note that Rice's Theorem not only bridges between semantic and syntactic aspects, but also in its statement displays a theme that is central in both computability and complexity theory: the study of which languages hard 
for which classes with respect to which types of reductions. This theme will also be important in the present paper.

"Rice's Theorem" is commonly used to refer both to the strong form just mentioned and the weaker form that speaks just of undecidability.

Theorem 1 (Rice's Theorem) Let $A$ be a nonempty, proper subset of the recursively enumerable sets. Then the language $\{M \mid L(M) \in A\}$ is $\mathrm{RE}-\leq_{m}$ hard or is coRE- $\leq_{m}$-hard.

Corollary 2 (Rice's Theorem, second version) Let $A$ be a nonempty, proper subset of the recursively enumerable sets. Then the language $\{M \mid L(M) \in A\}$ is undecidable.

Rice's Theorem may be viewed as a statement about the remarkable nontransparency of programs. Rice's Theorem says that no total Turing machine can test any nontrivial language property of programs.

Borchert and Stephan [BSO0] raise the question of whether complexitytheoretic analogs of Rice's Theorem hold. Rice's Theorem deals with recursively enumerable languages, and any such language is accepted by some Turing machine. Borchert and Stephan show that a related result holds for the case of boolean formulas and boolean circuits. Their result deals with counting properties of boolean formulas - those properties that depend solely on the number of satisfying assignments of a boolean formula. In particular, Borchert and Stephan prove that any nontrivial counting property of circuits is UP-hard. (Throughout this paper $\mathcal{C}$-hard always means $\mathcal{C}-\leq_{T}^{p}$-hard, unless some other reduction is explicitly inserted as in, for example, $\mathcal{C}-\leq_{1-t t}^{p}$-hardness.)

Hemaspaandra and Rothe [HR00] improve the UP-hardness lower bound of Borchert and Stephan [BS00] to UP $\mathrm{O}_{(1)}$-hardness. That is, they prove that every nontrivial counting property of circuits is $U P_{O(1)}$-hard. In the same vein, they ask if it is possible to improve the lower bound beyond $\mathrm{UP}_{O(1)}$, and they show that relativizable techniques cannot raise the UP $O(1)$-hardness lower bound to SPP-hardness, where SPP [OH93,FFK94] is the gap analog of UP. In particular, they note that if every nontrivial counting property of circuits is SPP-hard, then SPP $\subseteq \Delta_{2}^{p}$.

The class FewP, of Allender and Rubinstein [AR88], is the collection of all NP sets acceptable via polynomial-ambiguity nondeterminism; UP $\subseteq$ UP $O_{(1)} \subseteq$ FewP $\subseteq$ NP. We prove that every nontrivial counting property of circuits is FewP-hard (equivalently, is Few-hard), and indeed even is FewP- $\leq_{t t}^{p}$-hard and Few- $\leq_{t t}^{p}$-hard. We thus raise Hemaspaandra and Rothe's constant-ambiguity nondeterminism lower bound for nontrivial counting properties to polynomialambiguity nondeterminism. We prove that no relativizable technique can improve that lower bound to FewP- $\leq_{1-t t}^{p}$-hardness. We also prove an analog of Rice's Theorem for NP, namely, that all language properties of NP are NP$\leq_{m}^{p}$-hard or coNP- $\leq_{m}^{p}$-hard.

Due to space limitations, the proofs are omitted; they can be found in [HT02]. 


\section{Preliminaries}

This section presents the notation and definitions used in the paper. All sets, unless otherwise stated, are considered subsets of $\Sigma^{*}$, where $\Sigma$ is the standard alphabet $\{0,1\}$. The length of a string $x$ is denoted by $|x| . \Sigma^{n}$ denotes the set of strings in $\Sigma^{*}$ of length exactly $n$. For any $C$ and $n, C^{=n}=\{a|a \in C \wedge| a \mid=n\}$. We say that a set $A$ is a nontrivial subset of $B$ if $\emptyset \subsetneq A \subsetneq B .\langle\cdot, \ldots, \cdot\rangle$ usually denotes a standard, fixed, easily computable and invertible multi-arity pairing function (see [HHT97]) or a standard, fixed, easily computable and invertible 2-ary pairing function (which holds will be clear from context).

For any set $A, \chi_{A}$ denotes the characteristic function of $A$. That is, for any $x \notin A, \chi_{A}(x)=0$, and for any $x \in A, \chi_{A}(x)=1$. A boolean predicate $Q$ is a total function from $\Sigma^{*}$ to $\{0,1\}$. SAT denotes the set of all satisfiable boolean formulas. FP denotes the class of all (total) polynomial-time computable functions.

For any Turing machine $N$ and any $x \in \Sigma^{*}$, we will use $N(x)$ as an abbreviation for "the computation of $N$ on $x$." We will use DPTM as an abbreviation for "deterministic polynomial-time Turing machine." We will use NPTM as an abbreviation for "nondeterministic polynomial-time Turing machine." For any $\operatorname{NPTM} N, \# \operatorname{acc}_{N}$ is the function such that, for any $x \in \Sigma^{*}, \# \operatorname{acc}_{N}(x)$ is equal to the number of accepting computation paths of $N(x)$. We will use UPTM as an abbreviation for "unambiguous, nondeterministic polynomial-time Turing machine." That is, $N$ is a UPTM if and only if $N$ is an NPTM and, for all $x \in \Sigma^{*}$, the number of accepting paths of $N$ on input $x$ is at most 1 .

We now define some standard counting-based limited-ambiguity classes. We make use of the ambiguity-limited counting operator $\#_{g}$ [HR00] in defining these classes. In the definitions below, for simplicity of notation, we use $\#_{k}$ when we actually mean $\#_{\lambda x . k}$.

\section{Definition 3}

1 [Val79] \#P is the class of all functions $f: \Sigma^{*} \rightarrow \mathbb{N}$ such that there exists an NPTM $N$ such that, for all $x \in \Sigma^{*}$, the number of accepting paths of $N$ on input $x$ is exactly $f(x)$.

2 [HR00] For any total function $f: \mathbb{N} \rightarrow \mathbb{N}$, and for any complexity class $\mathcal{C}, \#_{\mathrm{f}} \cdot \mathcal{C}$ is the set of all functions $g: \Sigma^{*} \rightarrow \mathbb{N}$ such that there exist a language $L \in \mathcal{C}$ and a polynomial $p$ such that the following hold for each $x \in \Sigma^{*}$ :

(a) $g(x) \leq f(|x|)$, and

(b) $\|\{y|| y \mid=p(|x|) \wedge\langle x, y\rangle \in L\}\|=g(x)$.

3 [HR00] For each class $\mathcal{C}$, let \# $\#_{\text {const }} \cdot \mathcal{C}=\left\{g: \Sigma^{*} \rightarrow \mathbb{N} \mid(\exists k)\left[g \in \#_{k} \cdot \mathcal{C}\right]\right\}$.

4 [HV95] For each class $\mathcal{C}$, let

$$
\#_{\text {few }} \cdot \mathcal{C}=\left\{g: \Sigma^{*} \rightarrow \mathbb{N} \mid(\exists \text { polynomial } q)\left[g \in \#_{\mathrm{q}} \cdot \mathcal{C}\right]\right\}
$$


5 [Val76] UP $=\left\{L \mid\left(\exists g \in \#_{1} \cdot \mathrm{P}\right)\left(\forall x \in \Sigma^{*}\right)[x \in L \Longleftrightarrow g(x)>0]\right\}$.

6 [AR88] FewP $=\left\{L \mid\left(\exists g \in \#_{\text {few }} \cdot \mathrm{P}\right)\left(\forall x \in \Sigma^{*}\right)[x \in L \Longleftrightarrow g(x)>0]\right\}$.

$7[\mathrm{CH} 90] \mathrm{Few}=\mathrm{P}_{\text {fow }} \cdot \mathrm{P}[1]$, i.e., the class of languages accepted by $\mathrm{P}$ machines that on each input are allowed at most one query to a function from $\#_{\text {few }} \cdot \mathrm{P}$.

8 [OH93,FFK94] SPP is the class of all languages such that there exist a function $f \in \# \mathrm{P}$ and a polynomial-time computable function $g: \Sigma^{*} \rightarrow \mathbb{N}$ such that, for all $x$, the following hold:

(a) $x \notin L \Longrightarrow f(x)=g(x)$, and

(b) $x \in L \Longrightarrow f(x)=g(x)+1$.

FewP-hardness and Few-hardness are known to coincide (e.g., by using prefix search to pull down certificates one at a time, bit by bit, but note that doing so is truly using the adaptive nature of Turing reductions). FewP- $\leq_{t t}^{p}$-hardness and Few- $\leq_{t t}^{p}$-hardness are not known to coincide (and the "obvious" proof that they coincide, namely guessing all census values in parallel, does not seem to workinformally speaking, due to the fact that $\left(\begin{array}{c}q(n) \\ q(n) / 2\end{array}\right)$, where $q$ is a nonconstant polynomial, may be exponentially large), though certainly all Few- $\leq_{t t}^{p}$-hard sets are FewP- $\leq_{t t}^{p}$-hard.

We now define the standard reductions used in the paper.

Definition 4 Let $A$ and $B$ be arbitrary sets.

1 We say that $A \leq_{m} B$ ( $A$ recursively many-one reduces to $B$ ) if there exists a recursive function $\sigma$ such that, for all $x, x \in A$ if and only if $\sigma(x) \in B$.

2 We say that $A \leq_{T} B$ ( $A$ recursively Turing reduces to $B$ ) if there exists an oracle Turing machine $M$, such that $L\left(M^{B}\right)=A$ and, for each $x$, $M^{B}(x)$ halts.

3 We say that $A \leq_{m}^{p} B$ (A polynomial-time many-one reduces to $B$ ) if there exists a total, polynomial-time computable, function $\sigma$ such that, for all $x, x \in A$ if and only if $\sigma(x) \in B$.

4 We say that $A \leq_{T}^{p} B$ (A polynomial-time Turing reduces to $B$ ) if there exists an oracle DPTM $M$, such that $L\left(M^{B}\right)=A$.

5 We say that $A \leq_{t t}^{p} B$ ( $A$ polynomial-time truth-table reduces to $B$ ) if there exists a DPTM $M$ and a polynomial-time computable function $f$ such that, for any $x$, there exists an integer $m$ such that

(a) $f(x)=\left\langle q_{1}, q_{2}, \ldots q_{m}\right\rangle$, and

(b) $M\left(\left\langle x, \chi_{B}\left(q_{1}\right), \chi_{B}\left(q_{2}\right), \ldots, \chi_{B}\left(q_{m}\right)\right\rangle\right)$ accepts if and only if $x \in A$. 
6 For any $h: \mathbb{N} \rightarrow \mathbb{N}$, we say that $A \leq_{h(n)-t t}^{p} B$ (A polynomial-time $h(n)$ truth-table reduces to $B$ ) if there exists a DPTM $M$ and a polynomialtime computable function $f$ such that, for any $x$, there exists an integer $m \leq h(|x|)$ such that

(a) $f(x)=\left\langle q_{1}, q_{2}, \ldots q_{m}\right\rangle$, and

(b) $M\left(\left\langle x, \chi_{B}\left(q_{1}\right), \chi_{B}\left(q_{2}\right), \ldots, \chi_{B}\left(q_{m}\right)\right\rangle\right)$ accepts if and only if $x \in A$.

A set $B$ is $\mathcal{C}$-hard exactly if $(\forall C \in \mathcal{C})\left[C \leq_{T}^{p} B\right]$.

Next we present some notations about circuits and boolean formulas that will be used in the paper.

Definition 5 (see [BS00]) For any boolean formula (respectively, boolean circuits) $x, \#_{b}(x)$ (respectively, $\#_{c}(x)$ ) denotes the number of satisfying assignments of $x$ (respectively, the number of appropriate-length input bit vectors that make the output of the circuit 1).

In light of the existence of parsimonious versions of Cook's reduction (see [Gal74,Sim75]) and of efficient, parsimonious transformations between formulas and circuits, it holds that for each \#P function $f$ there exist functions $\hat{c}_{f} \in \mathrm{FP}$ and $\hat{b}_{f} \in \mathrm{FP}$ such that, for each $x, \hat{c}_{f}(x)$ is a boolean circuit satisfying $f(x)=\#_{c}\left(\hat{c}_{f}(x)\right)$ and $\hat{b}_{f}(x)$ is a boolean formula satisfying $f(x)=\#_{b}\left(\hat{b}_{f}(x)\right)$. For each $f \in \# \mathrm{P}$, arbitrarily choose one such $\hat{c}_{f}$ and one such $\hat{b}_{f}$ and denote these henceforward by $\hat{c}_{f}$ and $\hat{b}_{f}$.

We now present definitions related to the counting properties of circuits.

Definition 6 Let $A \subseteq \mathbb{N}$.

1 [BS00] Counting $(A)$ is the set of all boolean circuits such that the number of satisfying assignments of the circuit is a member of $A$. That is,

$$
\operatorname{Counting}(A)=\left\{\hat{c} \mid \#_{c}(\hat{c}) \in A\right\} \text {. }
$$

2 We say that $T_{0}(A)$ holds if and only if there exists an $n$ such that $n \in$ $A \Longleftrightarrow n+1 \notin A$, and the least such $n$ belongs to $\bar{A}$.

3 We say that $T_{1}(A)$ holds if and only if there exists an $n$ such that $n \in$ $A \Longleftrightarrow n+1 \notin A$, and the least such $n$ belongs to $A$.

4 For each $A \subseteq \mathbb{N}$, we say that $\operatorname{Counting}(A)$ is a counting property of circuits.

5 For each $\emptyset \subsetneq A \subsetneq \mathbb{N}$, we say that Counting $(A)$ is a nontrivial counting property of circuits.

Let $M_{1}, M_{2}, \ldots$ be any acceptable enumeration of Turing machines. The halting problem, which is RE- $\leq_{m}$-complete, is $\mathrm{HP}=\left\{x \mid M_{\operatorname{rank}(x)}(x)\right.$ halts $\}$, where $\operatorname{rank}(x)$ denotes the lexicographic rank of $x$, i.e., $\operatorname{rank}(\epsilon)=1, \operatorname{rank}(0)=$ $2, \operatorname{rank}(1)=3, \operatorname{rank}(00)=4$, etc. 


\section{3. $\mathrm{USAT}_{Q}$ and Hardness for Polynomial Ambiguity}

Hemaspaandra and Rothe [HR00] prove that every nontrivial counting property of circuits is $\mathrm{UP}_{O(1)}$-hard. They also prove that it is unlikely that the $\mathrm{UP}_{O(1)}$ lower bound can be raised much higher: If every nontrivial counting property of circuits is SPP-hard, then SPP $\subseteq \mathrm{P}^{\mathrm{NP}}$. (Fortnow [For97] provides a relativization in which SPP is not contained in $\mathrm{P}^{\mathrm{NP}}$.) In the light of these two results, it is natural to examine the complexity classes that fall between $\mathrm{UP}_{O(1)}$ and SPP, and to ask whether it is possible to raise the UP ${ }_{O(1)}$-hardness lower bound that holds for nontrivial counting properties.

Two natural complexity classes that lie between UP ${ }_{O(1)}$ and SPP are FewP and Few. FewP is the polynomial-ambiguity version of UP, and Few is the class of languages accepted by polynomial-ambiguity nondeterministic Turing machines operating under any polynomial-time computable counting acceptance mechanism (see [CH90] for full details, or see Definition 3 for a simple alternate definition/characterization of the class). It is known that $\mathrm{UP}_{O(1)} \subseteq$ FewP $\subseteq$ Few $\subseteq \mathrm{SPP}[\mathrm{KSTT} 92, \mathrm{FFK} 94]$.

In this section we prove that every nontrivial counting property of circuits is Few-hard, thus raising the lower bound. We first prove that for any nontrivial property $A$, there exists a predicate $Q$ such that at least one of Counting $(A)$ and Counting $(A)$ is $\leq_{m}^{p}$-hard for $\operatorname{USAT}_{Q}$, where, for any boolean predicate $Q$, $\mathrm{USAT}_{Q}$ is defined (see [VV86]) as follows.

$$
\chi_{\operatorname{USAT}_{Q}}(x)= \begin{cases}\chi_{\mathrm{SAT}}(x) & \text { if } \#_{b}(x) \in\{0,1\} \\ Q(x) & \text { otherwise. }\end{cases}
$$

The flavor of the following lemma, which we state here for completeness, is implicit in the comments at the end of Section 5.1 of [BS00].

Lemma 7 Let $A \subseteq \mathbb{N}$.

$1(\exists n, m: n<m)[n \notin A \wedge m \in A] \Longrightarrow(\exists Q)\left[\operatorname{USAT}_{Q} \leq_{m}^{p} \operatorname{Counting}(A)\right]$.

2 $(\exists n, m: n<m)[n \in A \wedge m \notin A] \Longrightarrow(\exists Q)\left[\overline{\mathrm{USAT}_{Q}} \leq_{m}^{p}\right.$ Counting $\left.(A)\right]$.

Glaßer and Hemaspaandra [GH00] prove that for every $Q, \operatorname{USAT}_{Q}$ is Few$\leq_{t t}^{p}$-hard.

Theorem 8 ([GH00]) If $L \in$ Few, then $(\forall Q)\left[L \leq_{t t}^{p} \operatorname{USAT}_{Q}\right]$.

We now can state the strengthening of the lower bound on the hardness of nontrivial counting properties of circuits from constant-ambiguity nondeterminism to polynomial-ambiguity nondeterminism.

Theorem 9 For any nontrivial $(\emptyset \subsetneq A \subsetneq \mathbb{N}) A$, Counting $(A)$ is Few- $\leq_{t t}^{p}$-hard (and thus certainly Few-hard, FewP-hard, and FewP- $\leq_{t t}^{p}$-hard).

We can prove Theorem 9 by noting that using Theorem 8 and Lemma 7 it follows; alternatively we can (and in the full version do) give a direct proof that 
gives more intuition about what is going on.

One might wonder whether it is possible to prove a strengthened version of Theorem 9 in which the "Few- $\leq_{t t}^{p}$ " in the statement of Theorem 9 is changed to "NP- $\leq_{T}^{p}$ " (or even to "NP- $\leq_{t t}^{p}$ "). In fact, as is essentially noted by Borchert and Stephan [BS00, p. 492], if such a strengthened claim were true then it would follow that NP $\leq_{T}^{p} \oplus \mathrm{P}$, where $\oplus \mathrm{P}[\mathrm{PZ83,GP86}]$ is the class of languages $L$ such that there exists a \#P function $f_{L}$ such that, for each $x \in \Sigma^{*}, x$ is in $L$ if and only if $f_{L}(x)$ is odd; and so since $\oplus \mathrm{P}$ is closed downward under Turing reductions [PZ83], if such a claim were true then NP $\subseteq$ P. However, Torán [Tor88,Tor91] constructed a relativized world in which NP is not contained in $\oplus \mathrm{P}$. Thus, Theorem 9's Few- $\leq_{t t}^{p}$-hardness lower bound, relativized in the natural way we will discuss in the next section, cannot be strengthened to NP- $\leq_{t t}^{p}$-hardness (or even to NP- $\leq_{T}^{p}$-hardness) using any relativizable technique.

We mention in passing that Theorem 9 seems neither to imply nor to be implied by a result of Borchert, Hemaspaandra, and Rothe that shows that certain "restricted counting classes" contain FewP [BHR00, Theorem 3.4]. On the one hand, the result of Borchert et al. applies only to promise classes; but on the other hand, the result of Borchert et al. (conditionally) concludes containment results rather than hardness results.

Valiant and Vazirani [VV86] prove that, for every $Q, \mathrm{USAT}_{Q}$ is $\leq_{\text {randomized }^{-}}^{p}$ hard for NP, where we are using $\leq_{\text {randomized }}^{p}$ to denote the Valiant-Vazirani ([VV86], see also [BS00]) notion of randomized reduction. So the following result (which is a more refined, detailed statement of the flavor of of [BSO0], Theorem 5.2) follows from Lemma 7.

Proposition 10 ([BS00]) Let $A \subseteq \mathbb{N}$.

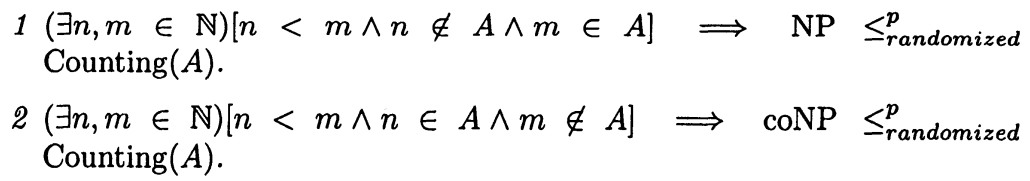

Proposition 10 gives a lower bound on the hardness of Counting $(A)$. It is thus natural to seek an interesting upper bound. Theorem 11 states that under certain assumptions, Counting $(A)$ is as easy as detecting unique solutions. In particular, for each nontrivial $A$, at least one of Counting $(A)$ and $\overline{\operatorname{Counting}(A)}$ $\leq_{m}^{p}$-reduces to $\mathrm{USAT}_{Q}$, for some $Q$.

Theorem 11 Let $A \subseteq \mathbb{N}$.

$$
\begin{aligned}
& 1 T_{0}(A) \Longrightarrow(\exists Q)\left[\operatorname{Counting}(A) \leq_{m}^{p} \operatorname{USAT}_{Q}\right] \\
& 2 T_{1}(A) \Longrightarrow(\exists Q)\left[\overline{\operatorname{Counting}(A)} \leq_{m}^{p} \operatorname{USAT}_{Q}\right]
\end{aligned}
$$

Corollary 12 For each $\emptyset \subsetneq A \subsetneq \mathbb{N}$, there exists a $Q$ such that at least one of Counting $(A)$ or $\overline{\operatorname{Counting}(A)} \leq_{m}^{p}$-reduces to $\mathrm{USAT}_{Q}$.

Lemma 7 proves that under suitable conditions Counting $(A)$ is $\leq_{m}^{p}$-hard 
for $\mathrm{USAT}_{Q}$, for some predicate $Q$. On the other hand, Theorem 11 proves that under suitable conditions, Counting $(A)$ is $\leq_{m}^{p}$-easy for $\operatorname{USAT}_{Q^{\prime}}$, for some predicate $Q^{\prime}$.

\section{A Relativized Upper Bound on the Complexity of Counting Properties}

Theorem 9 proves that all nontrivial counting properties of circuits are Few- $\leq_{t t}^{p}$-hard (and thus, FewP- $\leq_{t t}^{p}$-hard). Can the FewP- $\leq_{t t}^{p}$-hardness lower bound of nontrivial counting properties of circuits be improved? Hemaspaandra and Rothe [HR00] proved that raising the lower bound to SPP-hardness (a) would imply an unexpected complexity class containment, and (b) cannot be proven via relativizable proof techniques. However, in light of the fact that the previous UP-hardness and UP $O(1)$-hardness results in fact achieve in each case not just hardness (i.e., $\leq_{T}^{p}$-hardness) but even $\leq_{1-t t}^{p}$-hardness, it would be natural to hope that the FewP- $\leq_{t t}^{p}$-hardness result of Theorem 9 can at least be improved to FewP- $\leq_{1-t t}^{p}$-hardness. Nonetheless, we prove (in Theorem 17) that relativizable proof techniques cannot improve the FewP $-\leq_{t t}^{p}$-hardness lower bound of nontrivial counting properties to FewP $-\leq_{1-t t}^{p}$-hardness. In particular, we prove that there is a relativized world in which the following statement is false: "All nontrivial counting properties are FewP $-\leq_{1-t t}^{p}$-hard."

Before we state Theorem 17, we need to state what we mean by "counting property relative to an oracle." Counting, as defined and used in earlier sections, is based on the number of appropriate-length bit vectors that make the output of the circuit 1 . For the purpose of relativizing counting properties we will define and use another equivalent, easily relativizable version of counting based on the number of accepting paths of NPTMs. For any $A \subseteq \mathbb{N}$, we call this version of counting PathCounting $(A)$ and define it as follows. In what follows, let $N_{1}, N_{2}, \ldots$ be a fixed, nice enumeration of NPTMs such that, for each $x \in \Sigma^{*}, N_{i}$ on input $x$ robustly (i.e., for all oracles) runs within time $|x|^{i}+i$.

Definition 13 Let $A \subseteq \mathbb{N}$. Then $\operatorname{PathCounting}(A)$ is defined as follows.

$$
\text { PathCounting }(A)=\left\{\left\langle i, x, 1^{|x|^{i}+i}\right\rangle \mid \# \operatorname{acc}_{N_{i}}(x) \in A\right\} \text {. }
$$

It follows from the existence of parsimonious versions of Cook's reduction that, for any $A$, PathCounting $(A) \leq_{m}^{p}$ Counting $(A)$. It is also easy to see that, for any $A$, Counting $(A) \leq_{m}^{p}$ PathCounting $(A)$. It easily follows that, for each $A \subseteq \mathbb{N}$ and $B \subseteq \Sigma^{*}$, (a) $B \leq_{m}^{p} \operatorname{Counting}(A) \Longleftrightarrow B \leq_{m}^{p}$ PathCounting $(A)$, and (b) $B \leq_{T}^{p}$ Counting $(A) \Longleftrightarrow B \leq_{T}^{p}$ PathCounting $(A)$. In fact, the reductions can be chosen so as to be independent of $A$, as the following proposition notes.

Proposition 14 There exist polynomial-time computable functions $f$ and $g$ such that, for every $A$, the following hold.

$1\left(\forall x \in \Sigma^{*}\right)[x \in \operatorname{Counting}(A) \Longleftrightarrow f(x) \in \operatorname{PathCounting}(A)]$, and 
2 $\left(\forall x \in \Sigma^{*}\right)[x \in$ PathCounting $(A) \Longleftrightarrow g(x) \in$ Counting $(A)]$.

Next, we define the relativized version of PathCounting.

Definition 15 For each $B \subseteq \Sigma^{*}$ and each $A \subseteq \mathbb{N}$, we define PathCounting $^{B}(A)$ (PathCounting $(A)$ relative to oracle $\left.B\right)$ as follows.

$$
\operatorname{PathCounting}^{B}(A)=\left\{\left\langle i, x, 1^{|x|^{i}+i}\right\rangle \mid \# \operatorname{acc}_{N_{i}^{B}}(x) \in A\right\} .
$$

Since we will need it in the statement of Theorem 17, we explicitly state the definition of relativized truth-table reductions.

Definition 16 For any $h: \mathbb{N} \rightarrow \mathbb{N}$, and any $A, B, C \subseteq \Sigma^{*}, A \leq_{h(n)-t t}^{p, C} B(A$ polynomial-time $h(n)$-truth-table reduces to $B$ relative to oracle $C$ ) if there exists an oracle DPTM $M$ and a deterministic polynomial-time transducer $M_{1}$ such that, for all $x$, there exist an integer $m \in \mathbb{N}$ and strings $q_{1}, q_{2}, \ldots, q_{m}$ such that the following hold:

$1 m \leq h(|x|)$,

$2 M_{1}^{C}(x)$ produces $\left\langle q_{1}, q_{2}, \ldots q_{m}\right\rangle$ as its output, and

$3 M^{C}\left(\left\langle x, \chi_{B}\left(q_{1}\right), \chi_{B}\left(q_{2}\right), \ldots, \chi_{B}\left(q_{m}\right)\right\rangle\right)$ accepts if and only if $x \in A$.

Next we turn to the following result, which shows that relativizable proof techniques cannot improve the FewP $-\leq_{t t}^{p}$-hardness lower bound of nontrivial counting properties to FewP $-\leq_{1-t t}^{p}$-hardness.

Theorem 17 There is an oracle $B \subseteq \Sigma^{*}$ and a set $A, \emptyset \subsetneq A \subsetneq \mathbb{N}$, such that PathCounting ${ }^{B}(A)$ is not FewP $\mathrm{P}^{B}-\leq_{1-t t}^{p, B}$-hard.

The proof is by a diagonalization-based oracle construction involving counting and invoking the Party Lemma of Cai et al. [CGH+89].

\section{The Natural NP Analog of Rice's Theorem}

Rice's Theorem deals with language properties of RE sets. Borchert and Stephan [BS00] started the search for complexity-theoretic analogs of Rice's Theorem. They proved an analog of Rice's Theorem in circuit complexity that deals with the counting properties of circuits. In this section, we state an analog of Rice's theorem that deals with the language properties of NP. To be clear, let us specify more clearly our terminology. Let $N_{1}, N_{2}, \ldots$ be a fixed, nice enumeration of NPTMs. For specificity, let the enumeration be that of $\mathrm{Du}$ and Ko [DK00, Section 1.5] (though any effective enumeration of languages in NP in the formal time-sensitive sense of [DK00, Section 1.5] would work equally well). A property of NP is any subset of NP. A set $A \subseteq \mathbb{N}$ is said to be a language property of NP if there exists a property $\rho$ of NP such that $A=\left\{i \in \mathbb{N} \mid L\left(N_{i}\right) \in \rho\right\}$.

We prove that any nontrivial language property of NP sets is NP-hard. Note that this is, in some sense, the exact analog of Rice's Theorem for NP: 
Any nontrivial language property of NP is NP- $\leq_{m}^{p}$-hard or coNP- $\leq_{m}^{p}$-hard (compare this with Theorem 1).

Theorem 18 Let $A$ be any nonempty, proper subset of the NP sets. Then $\left\{i \mid L\left(N_{i}\right) \in A\right\}$ is NP- $\leq_{m}^{p}$-hard or is coNP $-\leq_{m}^{p}$-hard.

As an immediate corollary, we have the following result.

Corollary 19 Let $A$ be a nonempty, proper subset of the NP sets. Then $\{i \in$ $\left.\mathbb{N} \mid L\left(N_{i}\right) \in A\right\}$ is NP-hard.

Note that Theorem 18 is a natural complexity-theoretic analog of Theorem 1 and Corollary 19 is a natural complexity-theoretic analog of Theorem 2.

However are these two results trivial in light of the following fact which states that every nontrivial language property of NP is undecidable?

Fact 20 Let $N_{0}, N_{1}, \ldots$ be an enumeration of NPTMs. Let $A$ be a nontrivial subset of NP. Then $\left\{i \mid L\left(N_{i}\right) \in A\right\}$ is undecidable (in fact, is either RE- $\leq_{m}$ hard or coRE- $\leq_{m}$-hard).

It might seem that Theorem 18 follows from Fact 20. However, as we will show (as Theorem 23), under reasonable complexity-theoretic assumptions, RE$\leq_{m}$-hardness does not imply NP- $\leq_{m}^{p}$-hardness (though, in fact, under other complexity-theoretic assumption we will see that RE- $\leq_{m}^{p}$-hardness does imply NP- $\leq_{m}^{p}$-hardness). We first state a useful definition and result due to Karp and Lipton [KL80].

\section{Definition 21 ([KL80])}

1 For each language class $\mathcal{C}$ and each function $f: \mathbb{N} \rightarrow \mathbb{N}, \mathcal{C} / f$ is defined as follows. $\mathcal{C} / f=\left\{L \mid(\exists g)\left(\exists L_{1} \in \mathcal{C}\right)(\forall x)\left[\left|g\left(1^{|x|}\right)\right| \leq f(|x|) \wedge(x \in L \Longleftrightarrow\right.\right.$ $\left.\left.\left.\left\langle x, g\left(1^{|x|}\right)\right\rangle \in L_{1}\right)\right]\right\}$.

2 For each language class $\mathcal{C}$ and each function class $\mathcal{F}, \mathcal{C} / \mathcal{F}$ is defined as follows. $\mathcal{C} / \mathcal{F}=\{L \mid(\exists f \in \mathcal{F})[L \in \mathcal{C} / f]\}$.

Theorem $22[\mathrm{KL} 80]$ If $\mathrm{SAT} \in \mathrm{P} / O(\log n)$, then $\mathrm{P}=\mathrm{NP}$.

We now have the following result, which says that the issue of whether RE$\leq_{m}^{p}$-hardness implies NP-hardness is completely controlled by the $\mathrm{P}=\mathrm{NP}$ question.

\section{Theorem 23}

1 If $\mathrm{P}=\mathrm{NP}$, then every $\mathrm{RE}-\leq_{m}$-hard set is NP- $\leq_{m}^{p}$-hard (and thus certainly NP-hard).

2 If $\mathrm{P} \neq \mathrm{NP}$, then there is some $\mathrm{RE}-\leq_{m}$-hard set that is not $\mathrm{NP}$-hard (and thus certainly not NP- $\leq_{m}^{p}$-hard).

In this paper we have been discussing analogs, of a nonprobabilistic nature, 
of Rice's Theorem. We mention that one can investigate analogs of Rice's Theorem that use probabilistic notions in their attempts to frame analogs of Rice's Theorem $\left[\mathrm{BGI}^{+} 01\right]$ or that are aimed at handling probabilistic complexity classes [HT01].

\section{Conclusions and Open Issues}

This paper improved the lower bound for nontrivial counting properties of circuits from UP $\mathrm{U}_{(1)}$-hardness to Few-hardness. It showed that relativizable techniques cannot improve the Few-hardness lower bound of nontrivial counting properties of circuits to Few- $\leq_{1-t t}^{p}$-hardness. The paper also proved a Rice-style theorem for language properties of NP sets.

Can the Few- $\leq_{t t}^{p}$-hardness result of the present paper be improved to Few$\leq_{n^{k}-t t}^{p}$-hardness, for some fixed $k$ ? Or conversely, given an arbitrary $k$, does there exist a relativization - even stronger than the one mentioned earlier in the paper-such that there exists a nontrivial subset, $A$, of $\mathbb{N}$, such that $A$ is not

Few- $\leq_{n^{k}-t t}^{p}$-hard, or better yet, can one show some unexpected complexity class collapse that would follow were every nontrivial counting property of circuits Few- $\leq_{n^{k}-t t}^{p}$-hard? We conjecture that the Few- $\leq_{t t}^{p}$-hardness result cannot be improved to Few- $\leq_{n^{k}-t t}^{p}$-hardness.

Acknowledgments: We thank $\mathrm{H}$. Hunt for commenting that an alternate proof of Corollary 19 might be obtained using techniques similar to those of [Hun82], and we thank an anonymous IFIP TCS 2002 conference referee for helpful comments. This work was supported in part by grants NSF-CCR-9322513 and NSF-INT-9815095/DAAD-315-PPP-gü-ab.

\section{References}

[AR88] E. Allender and R. Rubinstein. P-printable sets. SIAM Journal on Computing, 17(6):1193-1202, 1988.

$\left[\mathrm{BGI}^{+} 01\right]$ B. Barak, O. Goldreich, R. Impagliazzo, S. Rudich, A. Sahai, S. Vadhan, and K. Yang. On the (im)possibility of obfuscating programs. Report 2001/069, Cryptology ePrint Archive, August 2001. Preliminary version appears in Advances in Cryptology - CRYPTO '2001.

[BHR00] B. Borchert, L. Hemaspaandra, and J. Rothe. Restrictive acceptance suffices for equivalence problems. London Mathematical Society Journal of Computation and Mathematics, 3:86-95, 2000.

[BS00] B. Borchert and F. Stephan. Looking for an analogue of Rice's Theorem in circuit complexity theory. Mathematical Logic Quarterly, 46(4):489$504,2000$.

[CGH $\left.{ }^{+} 89\right]$ J. Cai, T. Gundermann, J. Hartmanis, L. Hemachandra, V. Sewelson, K. Wagner, and G. Wechsung. The boolean hierarchy II: Applications. SIAM Journal on Computing, 18(1):95-111, 1989.

[CH90] J. Cai and L. Hemachandra. On the power of parity polynomial time. Mathematical Systems Theory, 23(2):95-106, 1990. 
[DK00] D. Du and K. Ko. Theory of Computational Complexity. John Wiley and Sons, 2000.

[FFK94] S. Fenner, L. Fortnow, and S. Kurtz. Gap-definable counting classes. Journal of Computer and System Sciences, 48(1):116-148, 1994.

[For97] L. Fortnow. Counting complexity. In L. Hemaspaandra and A. Selman, editors, Complexity Theory Retrospective II, pages 81-107. SpringerVerlag, 1997.

[Gal74] Z. Galil. On some direct encodings of nondeterministic Turing machines operating in polynomial time into P-complete problems. SIGACT News, 6(1):19-24, 1974.

[GH00] C. Glaßer and L. Hemaspaandra. A moment of perfect clarity I: The parallel census technique. SIGACT News, 31(3):37-42, 2000.

[GP86] L. Goldschlager and I. Parberry. On the construction of parallel computers from various bases of boolean functions. Theoretical Computer Science, 43(1):43-58, 1986.

[HHT97] Y. Han, L. Hemaspaandra, and T. Thierauf. Threshold computation and cryptographic security. SIAM Journal on Computing, 26(1):59-78, 1997.

[HR00] L. Hemaspaandra and J. Rothe. A second step towards complexitytheoretic analogs of Rice's Theorem. Theoretical Computer Science, 244(1-2):205-217, 2000.

[HT01] L. Hemaspaandra and M. Thakur. Rice-style theorems for complexity theory. Technical Report TR-757, Department of Computer Science, University of Rochester, Rochester, NY, September 2001.

[HT02] L. Hemaspaandra and M. Thakur. Lower bounds and the hardness of counting properties. Technical Report TR-768, Department of Computer Science, University of Rochester, Rochester, NY, January 2002.

[Hun82] H. Hunt. On the complexity of flowchart and loop program schemes and programming languages. Journal of the ACM, 29(1):228-249, 1982.

[HV95] L. Hemaspaandra and H. Vollmer. The Satanic notations: Counting classes beyond \#P and other definitional adventures. SIGACT News, 26(1):2-13, 1995.

[KL80] R. Karp and R. Lipton. Some connections between nonuniform and uniform complexity classes. In Proceedings of the 12th ACM Symposium on Theory of Computing, pages 302-309. ACM Press, April 1980. An extended version has also appeared as: Turing machines that take advice, L'Enseignement Mathématique, 2nd series, 28, 1982, pages 191-209.

[KSTT92] J. Köbler, U. Schöning, S. Toda, and J. Torán. Turing machines with few accepting computations and low sets for PP. Journal of Computer and System Sciences, 44(2):272-286, 1992.

[OH93] M. Ogiwara and L. Hemachandra. A complexity theory for feasible closure properties. Journal of Computer and System Sciences, 46(3):295325, 1993.

[PZ83] C. Papadimitriou and S. Zachos. Two remarks on the power of counting. In Proceedings 6th GI Conference on Theoretical Computer Science, pages 269-276. Springer-Verlag Lecture Notes in Computer Science \#145, January 1983. 
[Ric53] H. Rice. Classes of recursively enumerable sets and their decision problems. Transactions of the AMS, 74:358-366, 1953.

[Ric56] H. Rice. On completely recursively enumerable classes and their key arrays. Journal of Symbolic Logic, 21:304-341, 1956.

[Sim75] J. Simon. On Some Central Problems in Computational Complexity. $\mathrm{PhD}$ thesis, Cornell University, Ithaca, N.Y., January 1975. Available as Cornell Department of Computer Science Technical Report TR75-224.

[Tor88] J. Torán. Structural Properties of the Counting Hierarchies. PhD thesis, Universitat Politècnica de Catalunya, Barcelona, Spain, 1988.

[Tor91] J. Torán. Complexity classes defined by counting quantifiers. Journal of the $A C M, 38(3): 753-774,1991$.

[Val76] L. Valiant. The relative complexity of checking and evaluating. Information Processing Letters, 5(1):20-23, 1976.

[Val79] L. Valiant. The complexity of enumeration and reliability problems. SIAM Journal on Computing, 8(3):410-421, 1979.

[VV86] L. Valiant and V. Vazirani. NP is as easy as detecting unique solutions. Theoretical Computer Science, 47(3):85-93, 1986. 